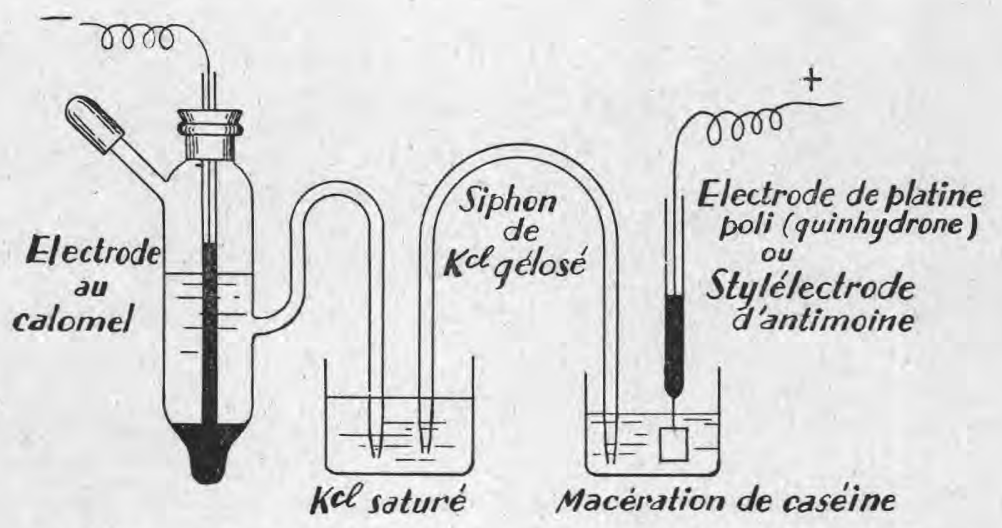

il en résulte une simplification du montage. Cette disposition permet en outre de n'utiliser que quelques centimètres cubes du liquide de macération et de déterminer très rapidement le $p \mathrm{H}$ d'une grande série d'échantillons.

Nous aurons l'occasion de revenir sur cette question pour montrer comment il convient d'interpréter les données fournies par cette technique dans la pratique du contrôle des caséines lactiques.

\title{
BIBLIOGRAPHIE
}

[1] J. Pien. Le Lait, t. XI, 1931, no 110, p. 1038.

[2] J. Pien et M. Bergier, Le Lait, t. XII, 1932, no 117, p. 640.

[3] J. Pien et S. Herschdoerfer. Le Lait, t. XIII, 1933, no 129, p. 1081.

[4] J. Pien et S. Hersohdoerfer. Le Lait, t. XIV, 1934, no 131, p. 30.

[5] J. Pien et M. Bergier. Le Lrit, t. XIV, 1934, no 132 , p. 155 et no 133 , p. 248.

[6] J. Pien, R. Martin et M. Bergier. Le Lait, t. XIV, 1934, no 134, p. 356 ; $\mathrm{n}^{\circ} 135$, p. $483 ; \mathrm{n}^{\circ} 136$, p. 582 .

[7] J. Pien, R. Martin et S. Herschdoerfer. Le Lait, t. XIV, 1934, no 137 , p. 709 .

[8] R. Dru. Le contrôle de la caséine-présure. XVII ${ }^{\mathrm{e}}$ Congrès de Chimie industrielle, Paris, 26 septembre et 3 octobre 1937.

\section{L'ORGANISATION DU CONTRÔLE HYGIÉNIQUE DU LAIT}

\author{
par le
}

\section{Dr G. BARTHÉLEMI}

Médecin-hygiéniste. M.R. San. I.

Dans le programme de rénovation du marché du lait ou pour mieux dire, dans la solution de cette importante question que constitue le problème du lait, il faut réserver une large part à 
l'organisation des services de contrôle, corollaire inévitable et nécessaire d'une législation, si l'on veut qu'elle soit respectée.

\section{BASES DU CONTROLE DU LAIT}

Pour autant que notre appréciation de la position du problème du lait en Belgique soit correcte, le lait constitue un produit accessoire de l'exploitation agricole puisqu'il ne fait qu'exceptionnellement l'objet des seules préoccupations de l'éleveur ou de l'agriculteur.

Et si, dans ces conditions, le producteur n'a aucun intérêt majeur à entourer la production du lait de toutes les garanties de sécurité qu'exigent nos connaissances scientifiques actuelles, il n'en reste pas moins, qu'en dernière analyse, le lait constitue une denrée alimentaire d'une importance capitale pour l'établissement du régime des nourrissons et pour le développement des enfants.

Le lait doit être considéré dans son but et non seulement dans ses moyens.

L'importance sociale et économique du lait, de par son influence sur la santé et le développement ultérieur des enfants, pour qui il constitue l'aliment essentiel, est trop connue pour y revenir davantage.

Mais nous insistons plutôt sur ee fait que la position du lait n'a pas encore été suffisamment définie du point de vue de l'hygiène.

Le lait est, avant toute autre chose, une denrée alimentaire et cette denrée intéresse toute l'enfance, tout l'avenir du pays.

C'est pourquoi le contrôle hygiénique doit s'adresser plus au "lait-aliment " qu'au "lait-produit agricole ».

Or, les dangers inhérents à la nature même de cet aliment résident dans le fait qu'il réalise un milieu de culture éminemment propice au développement de toutes les bactéries et, en particulier, les germes pathogènes pour l'être humain.

On ne peut méconnaître l'importance des ravages que causent encore en Belgique les affections gastro-intestinales chez les petits enfants soumis à l'allaitement artificiel.

Le lait en est le seul responsable et notre expérience personnelle nous en a fourni de nombreux exemples.

A cet égard, le producteur et le distributeur assument une responsabilité que couvre seule une négligence coupable dans l'inapplication des mesures élémentaires d'hygiène.

Que peut-il résulter d'autre, sinon une diminution de la valeur nutritive du lait et encore n'est-elle pas nocive, d'un écrémage partiel effectué à l'aide d'une écrémeuse lavée à l'eau bouillante ou d'un mouillage fait au moyen d'eau propre de la distribution communale? 
Or, une telle offense est sévèrement réprimée alors que le producteur qui fournit un lait renfermant couramment des millions de germes au centimètre cube et le distributeur qui le livre tel quel sont assurés d'une impunité absolue et combien dangereuse.

Qu'importe la persécution au densimètre, si le trayeur convalescent de fièvre typhoïde ou de diphtérie et le distributeur tuberculeux ou porteur de streptocoques hémolytiques continuent à répandre leurs bacilles autour d'eux, ou plus simplement encore si l'usage du savon et de l'eau est exclusivement réservé au dimanche dans la majorité de nos fermes !

Nous nous trouvons devant la triste réalité de devoir considérer, jusqu'à preuve du contraire, tous les laits comme bactériologiquement malpropres, et cela malgré les résultats favorables des analyses courantes (densité, sédiment, matière grasse, extrait sec dégraissé).

Il en résulte, du point de vue de l'hygiène, que la seule base logique du contrôle est le contrôle bactériologique, parce que le lait est un aliment d'une importance vitale. Il est moins dangereux de donner du lait mouillé à l'aide d'eau propre qu'un lait provenant d'un laitier malpropre, mais foncièrement honnête.

D'autre part, le contrôle bactériologique, qui est d'ailleurs d'application courante en Angleterre notamment où nous avons pu nous rendre compte des résultats qu'il permet d'obtenir, est le meilleur moyen d'apprécier, par étapes, les conditions dans lesquelles s'effectuent la production, le transport, le traitement et la distribution du lait.

Les prélèvements effectués à chacune de ces étapes donnent une indication certaine et précise des fautes commises dans les techniques qui s'y rapportent, alors que les expertises habituelles par les méthodes physiques et chimiques n'apprécient le lait que dans sa valeur intrinsèque momentanée.

Un résultat défavorable d'analyse bactériologique d'un lait déterminé permet d'en retrouver la cause aussi bien dans les récipients du distributeur que dans les conditions d'hygiène défectueuses de l'étable dont il provient.

Le contrôle bactériologique est également le seul qui permette de déclarer un lait propre ou impropre à la consommation alors qu'une fraude portant, par exemple, sur la teneur en matière grasse n'implique pas nécessairement que ce lait est nocif pour le consommateur, l'intérêt essentiel restant toujours celui de la Santé publique. Il est indispensable pour améliorer les conditions du marché du lait, parce qu'il est le seul sur lequel peuvent se baser les recherches effectuées pour perfectionner les techniques de production, de transport, de traitement et de distribution.

Quelle que soit la manière dont on envisage la question du lait, 
on ne doit jamais oublier que ce précieux et délicat liquide servira toujours d'aliment à un nourrisson, combien délicat et précieux !

\section{MODALITÉS DU CONTROLE HYGIÉNIQUE.}

Il est admis que le lait est la denrée alimentaire dont la falsification et l'adultération sont les plus aisées. Une répression suffisamment sévère peut en avoir raison dans le plus bref délai. Il suffit, à cet égard, que les pouvoirs des services de contrôle et les sanctions soient clairement définis dans la législation.

Mais, indépendamment des falsifications dont sont coupables des individus criminels bien plus que malhonnêtes, existent les altérations et surtout les contaminations accidentelles dont sont seules responsables la négligence et l'ignorance de leurs auteurs.

Celles-ci sont de tous les instants et surviennent à tout endroit de l'histoire du lait, depuis sa production jusqu'à sa distribution.

C'est pourquoi le contrôle du lait doit être unique et permanent.

Le prélèvement d'un échantillon à intervalles espacés ne constitue pas une sauvegarde pour la santé publique, parce que, dans ces conditions, il ne constitue plus qu'un risque aléatoire pour le laitier peu scrupuleux.

Le service de contrôle doit être suffisamment étendu pour tenir en éveil, par la fréquence de ses interventions (même quotidiennes à l'occasion), l'attention des intéressés.

En préconisant un contrôle unique, nous entendons qu'il doit être confié à un service jouissant de pouvoirs exclusivement et entièrement relatifs au lait. On ne peut donner à des services différents la charge de contrôler la production hygiénique du lait, car il en résulte fatalement des conflits de compétence et un manque de coordination fort préjudiciable aux intérêts à défendre.

Le principe de l'unicité du service de contrôle se justifie pour la raison qu'il n'y a pas de zones de démarcation entre les diverses étapes que doit suivre le lait. Toutes s'enchaînent les unes aux autres. La production, quels que soient les intérêts secondaires qu'elle suscite, ne peut pas être séparée de la distribution ou inversement; le traitement dépend directement du transport et sa technique doit s'inspirer des conditions de la production.

En conséquence, le service de contrôle doit être non seulement unique, mais aussi spécialisé. Nous nous réservons de justifier ce postulat ultérieurement.

\section{CARACTERE DU CONTROLE HYGIENIQUE, DU LAIT}

La mission du service de contrôle est de veiller à l'application des prescriptions légales et de rechercher les infractions. Son action est, par définition, répressive, mais elle ne peut se borner 
exclusivement, devant la complexité des éléments qui constituent le problème du lait, à faire appliquer des sanctions.

Si la répression est indispensable pour assurer le respect de la loi, l'éducation est d'égale valeur, elle lui est même supérieure.

Produire et vendre un lait sain n'est pas chose difficile, il suffit d'être propre et de travailler proprement. La difficulté réside dans le seul fait d'être propre au sens de "propreté : en ce qui concerne le lait.

Pour réaliser cette propreté, il faudrait exiger de toutes les personnes s'occupant du lait une éducation biologíque, bactériologique et technique pour laquelle elles ne sont pas préparées et qu'elles ne peuvent, en général, pas acquérir par défaut d'une formation de base suffisante.

Il appartient alors à des individus, spécialement éduqués et préparés en vue de cette mission, de conseiller et d'instruire les intéressés dans la manière de réaliser les conditions requises pour la production, le transport, le traitement et la distribution du lait et de les aider à remédier aux fautes de technique qu'ils sont exposés à commettre.

Nul n'est plus qualifié pour aider le laitier dans l'amélioration de ses procédés de travail que les membres du service de contrôle eux-mêmes, puisque leur compétence leur permet de trouver le remède dans le mal qu'ils ont dépisté. Le contrôle du lait doit done être surtout éducatif, il ne deviendra répressif qu'en cas de mauvais vouloir manifeste ou de négligence répétée. C'est pourquoi, une fois de plus, le service de contrôle doit être spécialisé.

\section{ORGANISATION DU CONTROLE}

L'organisation du contrôle du lait doit être envisagée sous les aspects suivants :

Technique du contrôle.

Choix du personnel.

Education du personnel.

Laboratoires d'analyse.

Centralisation du contrôle.

\section{Technique du contrôle}

Il n'intervient pas ici de discuter la valeur des diverses méthodes de contrôle physique, chimique et bactériologique du lait, ni de savoir quelles sont celles qui permettent d'apprécier avec le plus d'exactitude la qualité du lait et son degré de conformité avec les prescriptions légales.

Ce que nous en retenons est que la technique du contrôle doit être uniforme. 
L'exemple des pays étrangers, et en particulier de ceux qui sont déjà arrivés très loin dans la voie des réalisations pratiques, doit nous inspirer dans le choix des méthodes d'analyse considérées comme les plus efficaces et les plus exactes.

Mais le choix de la méthode étant fixé, il importe que la même méthode, ainsi officiellement consacrée, soit appliquée partout et suivant une technique réglée avec précision, afin que les résultats des analyses soient toujours eomparables entre eux et ne puissent prêter à équivoque en cas de contradiction.

L'usage de techniques d'analyse différentes fait trop souvent profiter du bénéfice du doute le fraudeur avéré.

L'uniformisation des méthodes et de la technique d'analyse n'est possible que grâce à l'emploi d'un matériel standardisé.

Ce vaste plan d'ensemble nous amène à envisager la création d'une Institution Centrale de Laiterie, de caractère purement technique et scientifique et administrée essentiellement dans un esprit d'hygiène. Cette limitation suffirait déjà amplement à son activité.

Dans une pareille institution, les "services techniques" sont chargés du contrôle technique et matériel des analyses ; ils assurent la standardisation du matériel instrumental en le contrôlant avant sa mise en usage, afin d'y apporter des corrections éventuelles.

Ils s'assurent de la qualité des produits utilisés comme réactifs et déterminent leur activité réactionnelle avant leur envoi aux laboratoires qui auront à les utiliser.

Ils procèdent à la préparation des produits nécessitant des soins particuliers ou pour lesquels la fabrication en grandes quantités offre seule des garanties suffisantes d'exactitude et d'uniformité : notamment les milieux de culture et certains réactifs chimiques.

Aucun de ces produits ne peut d'ailleurs être mis en usage avant d'être rigoureusement contrôlé tant au point de vue chimique qu'au point de vue bactériologique.

Les services techniques arrêtent également les modalités qui doivent présider à la prise des échantillons et déterminent les caractéristiques du matériel à utiliser à cet effet.

Les "services scientifiques », deuxième branche de l'activité de l'Institution Centrale de Laiterie, ont pour objet la recherche pure dans tous les domaines relatifs à la question du lait.

Ils confrontent les méthodes d'analyse en usage avec les découvertes récentes, ils mettent éventuellement au point des modifications ou simplifications utiles à apporter dans la technique du contrôle en particulier et dans la technique laitière en général ; car uniformité des méthodes ne veut pas dire adoption définitive 
d'une méthode déterminée ; celle-ci doit, au contraire, être réadaptée périodiquement suivant les progrès réalisés dans ce domaine.

Ils sont chargés de l'étude de toutes les questions soulevées dans la pratique laitière et que leur signalent les agents du service de contrôle, avec lesquels ils doivent travailler en collaboration étroite, tant pour rechercher les causes des anomalies observées que pour mettre au point le remède à $y$ apporter.

Ces investigations dans le domaine de la pratique laitière obligent les chercheurs à se tenir au courant des choses routinières de la laiterie et évitent de leur faire perdre de vue l'esprit dans lequel doivent être poursuivis leurs travaux de recherche.

Enfin, nous voulons réserver à l'Institution Centrale de Laiterie l'instruction et l'éducation pratique des agents du contrôle laitier, suivant un programme spécialisé que nous développerons plus loin.

L'Institution Centrale aura ainsi une triple mission :

a) Standardisation pratique.

b) Recherche scientifique.

c) Enseignement.

Quant à l'application proprement dite du contrôle, elle est faite par des laboratoires régionaux avec lesquels les agents du service de contrôle devront rester en contact permanent.

Ils effectuent les analyses par les méthodes élaborées ou contrôlées par l'Institution Centrale et au moyen du matériel contrôlé ou fourni par cette dernière.

Cette standardisation, répétons-le, est absolument indispensable pour permettre une figuration précise de la situation des ressources laitières, en ce qui concerne leur qualité, notamment :

a) La répartition géographique des laits suivant la composition chimique et les constantes physiques.

b) L'influence des conditions naturelles régionales sur la composition et la qualité (climat, alimentation, etc.).

c) L'influence des races laitières sur la composition au regard de leur distribution géographique; tous éléments nécessaires pour élaborer les bases d'une réglementation adéquate. Nous présumons que ces éléments font défaut encore actuellement.

Tout résultat douteux ou qui apparaît anormal est immédiatement transmis pour avis, avec tous les éléments d'appréciation recueillis, aux services scientifiques centraux, de même que toutes les difficultés sortant du domaine de la pratique courante du contrôleur sont également communiquées aux fins d'enquête.

Les laboratoires régionaux adressent, dans le plus bref délai, un protocole d'analyse à l'agent du contrôle dont émane l'échantillon en cause. Celui-ci se rend alors sur place et, tant par le raisonnement 
que par la persuasion, tâche d'obtenir du " coupable " l'amélioration des conditions qui ont provoqué l'intervention du service de contrôle.

Il est désirable, dans un but d'éducation et d'encouragement, que les bons résultats aussi bien que les mauvais soient régulièrement communiqués aux intéressés.

\section{Choix du personnel chargé du contrôie}

Il ressort de ce qui précède que le personnel auquel sera confié le contrôle du lait doit posséder des connaissances spéciales tant à cause des difficultés que présente la mission qu'il aura à remplir que du rôle d'éducateur qu'il aura à jouer.

Le problème du lait s'étendant à presque tous les domaines de la science, il est nécessaire que l'inspecteur du lait ait reçu une forte instruction moyenne, spécialement dans les branches des sciences naturelles, qui le rende apte à assimiler avec aisance la matière des cours spéciaux de laiterie et à subir avec succès l'épreuve terminale.

On devra exiger de l'inspecteur du lait de solides connaissances techniques et scientifiques dans le domaine de la laiterie ainsi qu'une formation générale suffisante pour lui permettre d'entretenir des relations faciles avec toutes les personnes intéressées à la question laitière, car il sera fatalement exposé à les rencontrer et à les heurter dans certains de leurs préjugés au cours de l'exercice de ses fonctions,

En plus de ses capacités intellectuelles, l'inspecteur du lait devra posséder des qualités morales qui feront de lui un agent de répression raisonnable et juste mais aussi un éducateur efficient.

Devant s'adresser à des individus chez qui la routine supplée généralement au manque de connaissances et de qui l'ignorance professionnelle n'a d'égale que la négligence, il devra posséder le tact, la patience et la force de persuasion indispensables pour mener à bien sa mission, ear c'est de la manière d'agir avee le producteur que dépend l'efficacité du rôle éducateur du service de contrôle.

A cet égard, la sélection du personnel de contrôle, en ce qui concerne ses qualités morales, aura autant de valeur que sa formation professionnelle.

\section{Education du personnel}

Les membres du personnel de l'Institution Centrale de Laiterie, spécialisés dans l'étude de chacun des sujets relatifs à la question laitière, constituent le corps professoral chargé d'initier les futurs inspecteurs à la connaissance de leurs devoirs et des pouvoirs que leur confère la législation (mise à jour). Les candidats acquièrent, par un stage dans les divers services centraux, ainsi que dans une 
ferme-laiterie expérimentale (à créer éventuellement), la formation technique et pratique qui constituera la base de leur activité.

Nous estimons que l'admission aux cours doit faire l'objet d'un examen préalable portant sur les matières de l'enseignement. moyen supérieur et plus spécialement sur les sciences naturelles.

D'autre part, l'examen terminal, du type concours, ne sera subi avec succès que si le candidat réunit un pourcentage déterminé des points, supérieur à la moyenne, afin d'obtenir un cadre dont l'instruction atteigne un niveau aussi élevé que possible.

Le programme des cours théoriques et pratiques, occupant en principe une année d'études, doit comprendre tous les domaines intéressant de près ou de loin le problème du lait et en retenir tous les éléments qui peuvent intervenir dans la tâche d'un inspecteur du lait.

Ce programme peut s'établir d'après le schéma suivant :

\section{A. Notions générales.}

Chimie. Physique, Hydro-géologie. Bactériologie relative au lait. Hygiène générale.

B. Anatomo-physiologie animale.

Eléments de nutrition. Entretien et hygiène de l'animal. Signes de santé et de maladie chez l'animal. Aspects, caractéristiques, conditions qui le rendent impropre à la consommation ou inapte à la production du lait.

C. Batiments,

Ferme, étable, laiterie, entrepôts, magasins. Principes de construction. Expertise sur plans. Hygiène générale. Affectation des locaux. Approvisionnement en eau : origine, qualité. Sources de contamination. Evacuation et destination des eaux résiduaires.

D. Equipement.

Matériel de laiterie. Récipients, effets sur le lait. Matériel et procédés de stérilisation.

E. Le Lait et ses produits.

Composition et ses variations. Caractéristiques. Appréciation sommaire. Techniques d'analyse et leur valeur. Classification.

F. Production.

Conditions essentielles pour la production hygiénique du lait. Méthodes de production. Dépistage des fautes.

G. Transport.

Procédés et conditions. 


\section{H. Traitement.}

Manutention. Pasteurisation : procédés, contrôle.

\section{Distribution.}

Conditions essentielles pour la conservation du lait avant la mise en vente et pour sa distribution. Conditionnement.

J. Altérations. - Falsifications.

Infection laitière. Conservateurs. Neutralisants. Colorants. Dépistage.

K. Inspection.

Technique et conditions de la prise d'échantillon. Méthodes pratiques et procédure d'inspection. Interprétation des rapports d'analyse chimique et bactériologique.

L. Personnel.

Hygiène du personnel s'occupant du lait. Compétence et aptitudes professionnelles.

\section{Législation.}

Lait. Epizooties. Denrées alimentaires. Maladies infectieuses. Interprétation. Pouvoirs. Administration. Application pratique. Fraudes. .

N. Organisation professionnelle.

Statistique. Tenue des documents. Rapports.

\section{Laboratoires de contrôle}

En vue de renforcer l'efficacité du contrôle du lait, nous estimons qu'il y a lieu de diviser le territoire qui lui est soumis en zones ou régions déterminées soit par les limites administratives existantes, soit par les limites géographiques naturelles, ces régions pouvant être des districts ruraux, des agglomérations ou des grandes villes.

Chacune de ces régions possède son unité de contrôle propre, constituée d'un laboratoire régional de contrôle et d'une équipe d'inspecteurs du lait dont l'effectif varie suivant l'importance respective de la production et de la consommation du lait dans la dite région.

Le laboratoire régional, possédant son propre personnel qualifié (chimistes, bactériologistes), jouit d'une indépendance relative dans son travail tout en restant sous l'autorité de l'Institution Centrale de Laiterie dans les conditions que nous avons précédemment décrites.

Quant aux inspecteurs du lait, la nature de leurs fonctions les tient en liaison permanente avec le laboratoire dont ils sont les aides bien plus que les subordonnés, car nous estimons qu'ils 
doivent être placés sous l'autorité directe du chef du service d'hygiène régional qui seul assume la responsabilité de la santé publique dans sa région, son district ou sa ville. (Nous entrevoyons, à l'instar de ce qui existe dans les autres pays, la création d'un cadre d'hygiénistes dans les attributions desquels rentrera la surveillance, à l'aide d'inspecteurs spécialisés, des denrées alimentaires, et nous maintenons notre point de vue, à savoir, que le problème du lait dans son ensemble est essentiellement un problème d'hygiène.)

La répartition des inspecteurs du lait par régions leur permet, dans leur champ d'action respectif, d'acquérir une connaissance approfondie des ressources laitières, des conditions d'exploitation souvent influencées par des habitudes locales, de la mentalité et du degré d'évolution des producteurs et des laitiers en général; elle leur permet de mieux connaître leurs "administrés " et de se faire connaître d'eux; elle doit, en résumé, faire naître, surtout chez le producteur, cette impression que l'inspecteur s'introduit chez lui plus en conseiller qu'en gendarme et aboutir à ce résultat que, en cas de difficulté, le producteur sollicitera l'intervention du contrôleur plutôt que de la redouter.

Nous partageons en effet complètement cette opinion des hygiénistes anglais que la vraie solution du problème du lait se trouve dans une collaboration étroite et harmonieúse entre le producteur et l'inspecteur spécialisé.

\section{Centralisation du contrôle}

Tous les résultats, quels qu'ils soient, bons ou mauvais, doivent être réunis à l'Institution Centrale de Laiterie afin de permettre à ses services d'en tirer tous enseignements utiles et de procéder à une classification générale des producteurs de lait suivant la qualité du produit qu'ils fournissent.

Ce répertoire systématique permet encore, en présence d'un résultat d'analyse défavoràble, de voir s'il constitue une exception dans le comportement habituel du producteur ou est un fait coutumier susceptible, après enquête, d'une investigation approfondie de ses méthodes de travail. Dans ce cas, et quand la bonne foi de l'intéressé ne peut être suspectée, le service de contrôle régional, dûment renseigné, disposera des moyens de porter remède aux défaillances techniques ou matérielles constatées, ou sinon appliquera les sanctions prévues par la législation.

De l'ensemble du matériel d'observation ainsi réuni se dégageront également des indications de nature à perfectionner les moyens de contrôle eux-mêmes et les méthodes de travail en usage à ce moment.

On pourra alors, connaissant exactement la position du problème 
du lait, envisager une nouvelle solution, celle de la création de catégories spéciales de lait pour lesquelles la production, le traitement et la distribution sont assujettis à l'octroi d'une licence et pour lesquelles les bases d'un contrôle, nécessairement spécial aussi, auront été solidement établies.

\section{CONCLUSIONS}

Ces réflexions, que nous suggère le contrôle hygiénique du lait, ne sont que l'expression, hâtivement résumée et forcément incomplète, de l'ensemble des observations que nous avons faites et des documents que nous avons réunis à l'occasion de plusieurs séjours à l'étranger, à l'effet d'y étudier précisément la question du lait.

C'est en déplorant le peu d'intérêt que les hygiénistes ont jusqu'à présent accordé à cette question que nous avons été amené à nous adonner à l'étude du problème du lait du point de vue de l'hygiène, car nous sommes pénétrés de cette notion que le problème du lait est avant tout un problème alimentaire et un problème de production.

A ce titre, l'hygiéniste, que sa formation a conduit dans les domaines les plus variés en relation avec la protection de la santé publique, est appelé à prendre une part active dans la solution de ce problème, car tout, dans la réalisation d'un approvisionnement en lait sain et propre, est affaire de prévention à l'origine.

Et l'essentiel réside dans la protection du lait contre l'infection microbienne, d'où qu'elle vienne.

Nous conclurons en faisant nôtres les réflexions émises au dernier Congrès d'hygiène à Southport, par le Dr J. A. FAIRER, et qui résument la question :

Il y trois facteurs à considérer :

(1. Le consommateur : qui croit obtenir un lait de bonne qualité alors qu'il n'en est rien.

2. Le laitier producteur : qui doit être éduqué et qui peut, lentement mais sûrement, apprendre comment réaliser et maintenir une production hygiénique du lait.

3. La vache laitière : qui n'a malheureusement aucune habitude de propreté ni aucune disposition naturelle à éviter le contact de ses déjections et qui requiert des soins et un entretien constants. "

(Laboratoire d'Hygiène de l'Université de Bruxelles.) 